\title{
Some Properties of Preopen Set in Closure Spaces
}

\author{
Halgwrd M. Darwesh ${ }^{1^{*}}$ Sarhad F.Namiq $^{2}$ \\ ${ }^{1}$ Department of Mathematics, College of Science, University of Sulaimani, 46001, Sulaimani, \\ Kurdistan Region, Iraq. \\ Email: halgwrd.darwesh@univsul.edu.iq \\ ${ }^{2}$ Department of Mathematics, College of education, University of Garmian, Kalar, Kurdistan \\ Region, Iraq. \\ Email: sarhad.faiq@garmian.edu.krd \\ * Corresponding author.
}

\begin{abstract}
Using the concept of preopen set, we introduce and study closure properties of pre-limit points, pre-derived sets, pre-interior and pre-closure of a set, preinterior points, pre-border, pre-frontier and pre-exterior in closure space. The relations between pre-closure of a set and pre-interior (point) in closure spaces and pre-closure of a set and pre-interior (point) in topological space are investigated.
\end{abstract}

Keywords: Pre-limit point, Pre-derived set, Pre-closure, Pre-interior points, Pre-border of sets, Pre- frontier of sets, Pre-exterior points.

\section{Introduction}

The notion of $(X, c)$ ( closure space) was introduced by Khampakdee [1]. He introduced open set and closed set in closure space [1]. And also he introduced Semi-open sets in biclosure spaces [2]. The notion of preopen set was introduced by Mashhour et al [3] . In [4] Halgwrd M. Darwesh defined preopen set in closure space which is different of preopen set in topological space, he introduced and studied some properties of preopen sets in closure space. They work in operation in topology in [5-28]. In this paper, we introduce the notions of pre-limit points, pre-derived sets, pre-interior of sets. We study

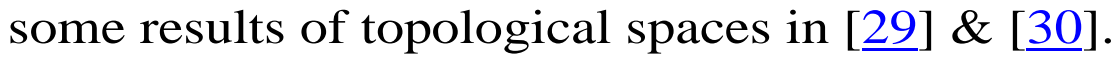

\section{Preliminaries}

Through this paper, $(X, \tau)$ (resp. $(X, c)$ ) always mean topological spaces (closure spaces). The intersection of all closed sets in topological spaces which contain $A$, is called closure of set denoted by $\operatorname{Cl}(A)$. And also the union of all open sets which contain in $A$ is called interior of $A$ which is denoted $\operatorname{Int}(A)$. A 
subset $A$ of $X$ is said to be preopen [ $\underline{3}$ ] if $A \subseteq \operatorname{Int}(\operatorname{Cl}(A))$. The complement of a preopen set is called a preclosed set.

\section{Definition 2.1 [1]}

A function $c: P(X) \rightarrow P(X)$ defined on the power set $P(X)$ of a set $X$ is called a closure operator on $X$ and the pair $(X, c)$ is called a closure space if the following axioms are satisfied:

(A1) $c(\phi)=\phi$.

(A2) $A \subseteq c(A)$ for every $A \subseteq X$.

(A3) $A \subseteq B \Rightarrow c(A) \subseteq c(B)$ for all $A, B \subseteq X$. A closure operator $c$ on a set $X$ is called additive (respectively, idempotent) if $A, B \subseteq X, c(A \cup B)=c(A) \cup$ $c(B)$ (respectively, for all $A \subseteq X \Rightarrow c c(A)=c(A)$. A subset $A \subseteq X$ is closed in the closure space $(X, c)$ if $c(A)=A$. It is called open, if its complement in $X$ is closed. The empty set and the whole space are both open and closed.

\section{Definition 2.2 [4]}

A subset $A$ of a space $(X, c)$ is said to be a preopen set, if there exists an open set $G$ such that $A \subseteq G \subseteq c(A)$. The complement of a preopen set is called preclosed. The family of all preopen sets denoted by $P O(X, C)$. The family of all preclosed sets denoted by $P C(X, c)$.

\section{Theorem 2.1 [4]}

A subset $A$ of a space $(X, c)$ is preclosed if and only if there exists a closed set $F$ such that $X \backslash c(X \backslash A) \subseteq F \subseteq A$.

\section{Proposition 2.1 [4]}

The union (intersection) of any family of preopen (preclosed) sets in a $\operatorname{space}(X, c)$ is preopen (preclosed).

\section{Definition 2.3 [4]}

The interior operator $i: P(X) \rightarrow P(X)$ corresponding to the closure operator $c$ on $X$ is given by; $i(A)=X \backslash c(X \backslash A)$.

\section{Theorem 2.2 [4]}

Let $A$ be a subset of a closure $(X, c)$. If $x \in c(A)$, then $G \cap A \neq \phi$, for each open subset $G$ of $X$ containing $x$. 


\section{Theorem 2.3 [4]}

Let $A$ be a subset of a closure $(X, c)$ and $c$ is idempotent on $X$, then $x \in c(A)$ if and only if $G \cap A \neq \phi$, for each open subset $G$ of $X$ containing $x$.

\section{Proposition 2.2 [4]}

Let $c$ be an idempotent closure operator on a set $X$. If $A$ is preopen in $X$ and $B \subseteq A \subseteq c(B)$, then $B$ is preopen.

\section{Theorem 2.4 [4]}

Let $c$ be an idempotent closure operator on $X$. A subset $A$ of $X$ is preopen if and only if $A \subseteq X \backslash c(X \backslash c A)=i c(A)$.

\section{Proposition 2.3 [4]}

If $A$ is closed and preopen in a space $(X, c)$, then $A$ is open.

\section{$3 \quad$ Some Properties of Preopen Sets}

\section{Definition 3.1}

Let $(X, c)$ be a closure space, $x \in X$ and $N$ be a subset of $X$. Then $N$ is called a preneighborhood of $x$ in $X$, if there exists a preopen set $V_{x}$ such that $x \in V_{x} \subseteq N$.

\section{Definition 3.2}

Let $A$ be a subset of a closure space $(X, c)$. A point $x \in X$ is said to be pre-limit point of $A$, if it satisfy the following assertion:

$V \cap(A \backslash\{x\}) \neq \phi$, for every preopen set $V$ such that $x \in V$.The set of all pre-limit points of $A$ is called the prederived set of $A$ and is denoted by $D_{P}(A)$.

Note that for a subset $A$ of $X$, a point $x \in X$ is not a pre-limit point of $A$ if and only if there exists a preopen set $V$ in $X$ such that $x \in V$ such that $V \cap(A \backslash\{x\})=\phi$, or (equivalently, $x \in V$ and $V \cap A=\phi$ or $V \cap A=\{x\}$ ).

\section{Theorem 3.1}

Let $c_{1}$ and $c_{2}$ be two closure operator on $X$ such that $P O\left(X, c_{1}\right) \subseteq P O\left(X, c_{2}\right)$. For any subset $A$ of $X$, every pre-limit point of $A$ with respect to $c_{2}$ is a pre-limit point of $A$ with respect to $c_{1 .}$

\section{Proof.}

Let $x$ be a pre-limit point of $A$ with respect to $c_{2}$. Then $V \cap(A\{x\}) \neq \phi$, for every preopen set $V$ with respect to $c_{2}$, such that $x \in V$. But $c_{1} \subseteq c_{2}$, so, in particular, $V \cap$ $(A\{x\}) \neq \phi$, for every preopen set $V$ with respect to $c_{1}$, such that $x \in V$. Hence $x$ is a pre-limit point of $A$ with respect to $c_{1}$. 
The converse of Theorem 3.1 is not true in general as seen in the following example.

\section{Example 3.1}

Let $X=\{a, b, c, d\}$ and defined closure operator $c_{1}: P(X) \rightarrow P(X)$ by:

$$
c_{1}(A)=\left\{\begin{array}{cc}
\phi & \text { if } A=\phi \\
\{b, c, d\} & \text { if } \phi \neq A \subseteq\{b, c, d\} \\
X & \text { otherwise }
\end{array}\right.
$$

So $P O\left(X, c_{1}\right)=\{\phi,\{a\},\{a, b\},\{a, c\},\{a, d\},\{a, b, c\},\{a, b, d\},\{a, c, d\}, X\}$.

And also defined closure operator $c_{2}: P(X) \rightarrow P(X)$ by:

$$
c_{2}(A)=\left\{\begin{array}{cl}
\phi & \text { if } A=\phi \\
X & \text { if } \phi \neq A \subseteq X .
\end{array}\right.
$$

So $P O\left(X, c_{2}\right)=P(X)$. We have $P O\left(X, c_{1}\right) \subseteq P O\left(X, c_{2}\right)$. Let $A=\{a, b\}$, then $D_{P}(A)=$ $\{b, c, d\}$ with respect $P O\left(X, c_{1}\right)$ and $D_{P}(A)=\phi$ with respect $P O\left(X, c_{2}\right)$. Note that $d$ is pre-limit point of $A$ with respect $P O\left(X, c_{1}\right)$, but it is not a pre-limit point of $A$ with respect $P O\left(X, c_{2}\right)$.

\section{Theorem 3.2}

For any subsets $A$ and $B$ of $(X, C)$, the following assertions are valid:

(1) If $A \subseteq B$, then $D_{P}(A) \subseteq D_{P}(B)$.

(2) $D_{P}(A) \cup D_{P}(B) \subseteq D_{P}(A \cup B)$.

(3) $D_{P}(A \cap B) \subseteq D_{P}(A) \cap D_{P}(B)$.

(4) $D_{P}\left(D_{P}(A)\right) \backslash A \subseteq D_{P}(A)$.

(5) $D_{P}\left(A \cup D_{P}(A)\right) \subseteq A \cup D_{P}(A)$.

Proof. (1): Let $x \in D_{P}(A)$. Then $x \in X$ is a pre-limit point of $A$. So $V \cap$

$(A \backslash\{x\}) \neq \phi$, for every preopen set $V$. But $A \subseteq B$, so $V \cap(B \backslash\{x\}) \neq \phi$, then $x$ is a pre-limit point of $B$, that is $x \in D_{P}(B)$. Hence, $D_{P}(A) \subseteq D_{P}(B)$.

(2) We have $A \subseteq A \cup B$ and $B \subseteq A \cup B$, then by(1) $D_{P}(A) \subseteq D_{P}(A \cup B)$ and $D_{P}(B) \subseteq D_{P}(A \cup B)$. Hence, $D_{P}(A) \cup D_{P}(B) \subseteq D_{P}(A \cup B)$.

(3) We have $A \cap B \subseteq A$ and $A \cap B \subseteq B$, then by(2) $D_{P}(A \cap B) \subseteq D_{P}(A)$ and $D_{P}(A \cap B) \subseteq D_{P}(B)$. Hence, $D_{P}(A \cap B) \subseteq D_{P}(A) \cap D_{P}(B)$.

(4) Let $x \in D_{P}\left(D_{P}(A)\right) \backslash A$. So $x \in D_{P}\left(D_{P}(A)\right)$ and $x \notin A$. Then, $x$ is a pre-limit point of $D_{P}(A)$. That is, $V \cap\left(D_{P}(A) \backslash\{x\}\right) \neq \phi$, for every preopen set $V$. Then, 


\section{كرميان}

there exists $y \in V \cap\left(D_{P}(A) \backslash\{x\}\right)$. So $y \in V$ and $y \in D_{P}(A) \backslash\{x\}$. Then, $y \in$ $D_{P}(A)$ and $y \neq x$. Thus, $y$ is a pre-limit point of $A$. Then, $V \cap(A \backslash\{y\}) \neq \phi$ and $y \neq x$. If we take $z \in V \cap(A \backslash\{y\})$, so $x \neq z$ because $x \notin A$. Hence, $V \cap$ $(A \backslash\{x\}) \neq \phi$, then $x$ is a pre-limit point of $A$. Therefore $x \in D_{P}(A)$. Thus $D_{P}\left(D_{P}(A)\right) \backslash A \subseteq D_{P}(A)$.

(5) Let $x \in D_{P}\left(A \cup D_{P}(A)\right)$. Then $x$ is a pre-limit point of $A \cup D_{P}(A)$. If $x \in A$, the result is obvious. Assume that $x \notin A$. Then, $V \cap\left(A \cup D_{P}(A) \backslash\{x\}\right) \neq \phi$, for all preopen set $V$.This means that, $V \cap(A \backslash\{x\}) \neq \phi$ or $V \cap\left(D_{P}(A) \backslash\{x\}\right) \neq \phi$. The first case implies $x \in D_{P}(A)$. If $V \cap\left(D_{P}(A) \backslash\{x\}\right) \neq \phi$, then $x \in D_{P}\left(D_{P}(A)\right)$. Since $x \notin A$, it follows similarly from (4)that $x \in D_{P}\left(D_{P}(A)\right) \backslash A \subseteq D_{P}(A)$. Therefore (5) is valid.

In general, neither inclusion of Theorem 3.2 is true as we will seen in the following examples. 


\section{Example 3.2}

Let $X=\{a, b, c, d\}$ and defined closure operator $c: P(X) \rightarrow P(X)$ by: $c(A)=$

$$
\left\{\begin{array}{c}
A \quad \text { if } A \in\{\phi,\{b\},\{c\},\{b, c\}\} \\
\{a, b\} \quad \text { if } A \in\{\{a\},\{a, b\}\} \\
\{c, d\} \quad \text { if } A \in\{\{d\},\{c, d\}\} \\
\{a, b, c\} \text { if } A \in\{\{a, c\},\{a, b, c\}\} \\
\{b, c, d\} \quad \text { if } A \in\{\{b, d\},\{b, c, d\}\} \\
X \quad \text { otherwise }
\end{array}\right.
$$

Hence $P O(X, c)=\{\phi,\{a\},\{d\},\{a, b\},\{a, d\},\{c, d\},\{a, b, d\},\{a, c, d\}, X\}$. For two subsets $A=\{a, c\}$ and $B=\{a, b, d\}$ of $X$, we get $D_{P}(A)=\{b\} \subseteq\{b, c\}=D_{P}(B)$, but $A \nsubseteq B$. This shows that the converse of Theorem 3.2(1) is not valid.

\section{Example 3.3}

Let $X=\{a, b, c, d, e\}$ and defined closure operator $c: P(X) \rightarrow P(X)$ by:

$$
c(A)=\left\{\begin{array}{cc}
A & \text { if } A \in\{\phi\}=\mathcal{F} \\
\{b, e\} & \text { if } A \in\{\{b\},\{e\},\{b, e\}\}=\mathcal{G} \\
\{a, b, e\} & \text { if } A \in\{\{a\},\{a, e\},\{a, b\},\{a, b, e\}\}=\mathcal{H} \\
\{b, c, d, e\} & \text { if } A \notin\{\mathcal{F}, \mathcal{G}, \mathcal{H}\} \text { and } A \subsetneq\{b, c, d, e\} \\
X & \text { otherwise }
\end{array}\right.
$$

$P O(X, c)=\{\phi,\{a, b\},\{a, c\},\{a, d\},\{c, d\},\{a, b, c\},\{a, b, d\},\{a, c, d\},\{a, c, e\}$,

$\{a, d, e\},\{a, b, c, d\},\{a, b, c, e\},\{a, b, d, e\},\{a, c, d, e\}, X\}$. Now consider two subsets $A=\{a, b\}$ and $B=\{b, c, d\}$ of $X$. Then $D_{P}(A)=\{b, e\}, D_{P}(B)=\{a, e\}$, and so $D_{P}(A \cap B)=\phi$, but $D_{P}(A) \cap D_{P}(B)=\{e\} \nsubseteq \phi=D_{P}(A \cap B)$. Thus the equality in Theorem 3.2 (5)is not valid.

\section{Example 3.4}

Let $X=\{a, b, c, d\}$ and defined closure operator $c: P(X) \rightarrow P(X)$ by:

$$
c(A)= \begin{cases}A & \text { if } A \in\{\phi,\{a\},\{d\},\{a, d\}\} \\ X & \text { otherwise }\end{cases}
$$

Hence

$P O(X, c)=$

$\{\phi,\{b\},\{c\},\{a, b\},\{a, c\},\{b, c\},\{b, d\},\{c, d\},\{a, b, c\},\{a, b, d\},\{a, c, d\},\{b, c, d\}, X\}$. 
Let $A=\{a, b\}$ and $B=\{a, c\}$ be subsets of $X$. Then $D_{P}(A)=\phi=D_{P}(B) . D_{P}(A) \cup$ $D_{P}(B)=\phi, D_{P}(A \cup B)=\{a, d\}$ but $D_{P}(A \cup B)=\{a, d\} \nsubseteq \phi=D_{P}(A) \cup D_{P}(B)$. Thus the equality in Theorem 3.2(2) Error! Reference source not found. is not valid. For a subset $A=\{a, b, c\}$ of $X$, we have $D_{P}\left(D_{P}(A)\right)=D_{P}(\{a, d\})=\phi$. But $D_{P}(A)=$ $\{a, d\} \nsubseteq D_{P}\left(D_{P}(A)\right) \backslash A=\phi$, and so the equality in Theorem 3.2(4) is not valid. Now for a subset $B=\{b, c\}$ of $X$, we get $D_{P}(B)=\{a, d\}$, and so $B \cup D_{P}(B)=X$ and $D_{P}(X)=$ $\{a, d\}$, but $B \cup D_{P}(B)=X \nsubseteq D_{P}\left(B \cup D_{P}(B)\right)=\{a, d\}$. This shows that $D_{P}(A \cup$ $\left.D_{P}(A)\right)=A \cup D_{P}(A)=X$. Hence the equality in Theorem 3.2(5) is not valid.

\section{Definition 3.3}

Let $(X, C)$ be a closure space and $A \subseteq X$. The intersection of all preclosed sets containing $A$ is called the pre-closure of $A$, denoted by $C l_{P}(A)$.

\section{Theorem 3.3}

Let $(X, c)$ be a closure space, $A, B \subseteq X$ then the following properties are true:

(1) $C l_{P}(A)$ is preclosed set.

(2) $A \subseteq C l_{P}(A)$.

(3) $C l_{P}(A)$ is smallest preclosed set which containing $A$.

(4) If $A \subseteq B$, then $C l_{P}(A) \subseteq C l_{P}(B)$.

(5) $C l_{P}(A) \cup C l_{P}(B) \subseteq C l_{P}(A \cup B)$.

(6) $C l_{P}(A \cap B) \subseteq C l_{P}(A) \cap C l_{P}(B)$.

(7) $A$ is preclosed set if and only if $A=C l_{P}(A)$.

(8) $C l_{P}\left(C l_{P}(A)\right)=C l_{P}(A)$.

\section{Proof:}

1.It follows from Definition 3.3 and Proposition 2.1.

2. Obvious.

3. From (1) and (2), we get $C l_{P}(A)$ is preclosed set which containing $A$. It is enough to show $C l_{P}(A)$ is smallest preclosed. Let $L$ be any preclosed set with $A \subseteq L$. Then, $L$ is one of the preclosed sets in which the intersection is taken it is mean $\operatorname{Cl}_{P}(A)=$ $\bigcap\{K, K$ is preclosed set and $A \subseteq K\}$. Hence $C l_{P}(A)$ is the smallest preclosed set containing $A$.

4. BY (2) $B \subseteq C l_{P}(B)$, since $A \subseteq B$, so $A \subseteq C l_{P}(B)$, but $\boldsymbol{C l}_{P}(A)$ is the smallest preclosed set containing $A$. So $C l_{P}(A) \subseteq C l_{P}(B)$. 
5. It follows from (4).

6. It follows from (4).

7. Let $A=C l_{P}(A)$. Since $C l_{P}(A)$ is preclosed set by (1), then $A$ is a preclosed set. Conversely: Let $A$ be a preclosed set. Then $A$ is the smallest preclosed set which contains $A$. So $A=C l_{P}(A)$ by (3).

8. Let $L=C l_{P}(A)$. So $L$ is a preclosed set by (1), then by (7) $L=C l_{P}(L)$. Thus $C l_{P}(A)=$ $C l_{P}\left(C l_{P}(A)\right)$.

\section{Theorem 3.4}

Let $(X, c)$ be a closure space and $A \subseteq X$. Then $x \in C l_{P}(A)$ if and only if $A \cap V \neq \phi$, for all preopen set $V$ which contains $x$.

\section{Proof.}

Let $x \in C l_{P}(A)$ and suppose that $A \cap V=\phi$, for some preopen set $V$ which contains $x$. This implies that $X / V$ is a preclosed set and $A \subseteq X / V$. So $C_{P}(A) \subseteq C l_{P}(X / V)=$ $X / V$. This implies that $x \in X / V$, which is a contradiction. Therefore, $A \cap V \neq \phi$, for all preopen set $V$, Which contains $x$.

Conversely. If $x \notin C l_{P}(A)$, then there exists a preclosed set $K$ such that $A \subseteq K$ and $x \notin K$. Hence $X \backslash K$ is a preopen set which containing $x$ and $A \cap(X \backslash K) \subseteq A \cap(X \backslash A)$ $=\phi$. Which is a contradiction. Hence, $x \in C l_{P}(A)$ is valid.

\section{Corollary 3.1}

For any subset $A$ of a closure space $(X, c)$, we have $D_{P}(A) \subseteq C l_{P}(A)$.

\section{Proof.}

Let $x \in D_{P}(A)$. Then, $A /\{x\} \cap V \neq \phi$, for all preopen set $V$ which contains $x$. So $A \cap V \neq \phi$, for all preopen set $V$ that contains $x$. Thus, by Theorem $3.4 x \in C l_{P}(A)$.

\section{Theorem 3.5}

For any subset $A$ of a closure space $(X, c)$, we have $C l_{P}(A)=A \cup D_{P}(A)$.

\section{Proof.}

Let $x \in C l_{P}(A)$. Assume that $x \notin A$ and let $V$ be a preopen set with $x \in V$. Then, $A$ / $\{x\} \cap V \neq \phi$, and so $x \in D_{P}(A)$. Hence $C l_{P}(A) \subseteq A \cup D_{P}(A)$. The reverse inclusion is valid by $A \subseteq C l_{P}(A)$ and Corollary 3.1.

\section{Theorem 3.6}


For a subset $A$ of a closure space $(X, c)$, we have $A$ is preclosed if and only if $D_{P}(A)$ $\subseteq A$.

\section{Proof.}

Assume that $A$ is preclosed. Let $x \notin A$, i.e., $x \in X \backslash A$. Since $X \backslash A$ is preopen, so $x$ is not a pre-limit point of $A$, i.e., $x \notin D_{P}(A)$, because $(X \backslash A) \cap(A \backslash\{x\})=\phi$. Hence, $D_{P}(A) \subseteq A$. The reverse implication is followed by Theorem 3.5.

\section{Corollary 3.2}

Let $A$ be a subset of a closure space $(X, c)$. If $F$ is a preclosed superset of $A$, then $D_{P}(A) \subseteq F$.

\section{Proof.}

By Theorem 3.2 (1) and Theorem 3.6, $A \subseteq F$ implies $D_{P}(A) \subseteq D_{P}(F) \subseteq F$.

\section{Theorem 3.7}

Let $A$ and $\mathrm{B}$ be any subsets of a closure space $(X, c)$ such that $A$ is preopen. If the family of all preopen subsets of $X$ is form a topology on $X$, then $A \cap C l_{P}(B) \subseteq$ $C l_{P}(A \cap B)$.

\section{Proof.}

Let $x \in A \cap C l_{P}(B)$. Then, $x \in A$ and $\in C l_{P}(B)=B \cup D_{P}(B)$. If $x \in B$, then $x \in A \cap$ $B \subseteq C l_{P}(A \cap B)$. If $x \notin B$, then $x \in D_{P}(B)$ and so $B /\{x\} \cap V \neq \phi$, for all preopen set $V$ containing $x$. Since $A$ is preopen and $V \cap A$ is also a preopen set containing $x$. Hence, $V \cap(A \cap B)=(V \cap A) \cap B \neq \phi$, and consequently $x \in C l_{P}(A \cap B)$. Therefore, $A \cap C l_{P}(B) \subseteq C l_{P}(A \cap B)$.

\section{Example 3.5}

Let $X=\{a, b, c, d\}$ and defined closure operator $c: P(X) \rightarrow P(X)$ by:

$$
c(A)=\left\{\begin{array}{cl}
A & \text { if } A \in\{\phi,\{a\}\}=\mathcal{F} \\
\{a, d\} & \text { if } A \in\{\{d\},\{a, d\}\}=\mathcal{G} \\
\{a, c, d\} & \text { if } A \notin\{\mathcal{F}, \mathcal{G}\} \text { and } A \subseteq\{a, c, d\} \\
X & \text { otherwise }
\end{array} .\right.
$$

Hence $P O(X, c)=\{\phi,\{b\},\{a, b\},\{b, c\},\{b, d\},\{a, b, c\},\{a, b, d\},\{b, c, d\}, X\}$

which is a topology on $X$. Consider the subset $A=\{a, b\}$ and $B=\{b, c\}$ of $X$, then $A \cap$ 
$C l_{P}(B)=\{a, b\} \neq X=C l_{P}(A \cap B)$. This shows that the equality in Theorem 3.7 is not true in general.

\section{Example 3.6}

The family of all preopen subsets of Example 3.4 does not form a topology on $X$ and since for subsets $A=\{a, b\}$ and $B=\{b, c\}$ of the closure space $X A \cap C l_{P}(B)=$ $\{a, b\} \nsubseteq\{b\}=C l_{P}(A \cap B)$. This shows that the conditions that the family of all preopen sets of $X$ form a topology, in Theorem 3.6 is necessary and it can not be dropped.

\section{Definition 3.4}

A closure space $(X, c)$ is said to be discrete if every subset of $X$ is open set.

\section{Note that}

(1) An closure spaces $(X, c)$ is discrete if and only if every subset of $X$ is closed.

(2) If $A$ is a subset of a discrete closure space $(X, c)$, then $D_{P}(A)=\phi$.

\section{Proposition 3.1}

Let $A$ be a subset of a closure space $(X, c)$. If a point $x \in X$ is a pre-limit point of $A$, then $x$ is also a pre-limit point of $A \backslash\{x\}$.

Proof. Obvious.

\section{Definition 3.5}

Let $A$ be a subset of a closure space $(X, c)$. A point $x \in X$ is called a pre-interior point of $A$, if there exists a preopen set $V$ such that $x \in V \subseteq A$. The set of all pre-interior points of $A$ is called the pre-interior of $\mathrm{A}$ and is denoted by $\operatorname{Int}_{P}(A)$.

\section{Proposition 3.2}

For subsets $A$ and $B$ a closure space $(X, c)$, the following assertions are valid.

(1) $\operatorname{Int}_{P}(A)$ is the union of all preopen subsets of $A$.

(2) $\operatorname{Int}_{P}(A)$ is the largest preopen set contained in $A$.

(3) $A$ is preopen if and only if $A=\operatorname{Int}_{P}(A)$.

(4) $\operatorname{Int}_{P}\left(\operatorname{Int}_{P}(A)\right)=\operatorname{Int}_{P}(A)$.

(5) $\operatorname{Int}_{P}(A)=A \backslash D_{P}(X \backslash A)$.

(6) $X \backslash \operatorname{Int}_{P}(A)=C l_{P}(X \backslash A)$.

(7) $X \backslash C l_{P}(A)=\operatorname{Int}_{P}(X \backslash A)$.

(8) If $A \subseteq B$, thenInt $\operatorname{In}_{P}(A) \subseteq \operatorname{Int}_{P}(B)$.

(9) $\left.\operatorname{Int}_{P}(A) \cup \operatorname{Int}_{P}(B)\right) \subseteq \operatorname{Int}_{P}(A \cup B)$.

(10)

$$
\operatorname{Int}_{P}(A \cap B) \subseteq \operatorname{Int}_{P}(A) \cap \operatorname{Int}_{P}(B) .
$$




\section{Proof:}

1.Let $\left\{V_{i}: i \in \Lambda\right\}$ be the collection of all preopen subsets of $X$ contained in $A$. If $x \in$ $\operatorname{Int}_{P}(A)$, then, there exists $j \in \Lambda$ such that $x \in V_{j} \subseteq A$. Hence, $x \in \bigcup_{i \in \Lambda} V_{i}$, and so $\operatorname{Int}_{P}(A) \subseteq \bigcup_{i \in \Lambda} V_{i}$. On the other hand, if $y \in \bigcup_{i \in \Lambda} V_{i}$, then $y \in V_{k} \subseteq A$ for some $k \in \Lambda$. Thus, $y \in \operatorname{Int}_{P}(A)$, and so $\bigcup_{i \in \Lambda} V_{i} \subseteq \operatorname{Int}_{P}(A)$. Accordingly, $\operatorname{Int}_{P}(A)=\bigcup_{i \in \Lambda} V_{i}$.

2. Since $\operatorname{Int}_{P}(A)=\bigcup_{G \subseteq A}\{G, G$ is preopen set $\}$, so by Proposition $2.1 \operatorname{Int}_{P}(A)$ is a preopen set. Also $\operatorname{Int}_{P}(A) \subseteq A$. Now, to prove $\operatorname{Int}_{P}(A)$ is the largest preopen set contained in $A$. Let $H$ be any other preopen set that contained in $A$. Since $H$ is a preopen set and $H \subseteq A$. So $H \subseteq \bigcup_{G \subseteq A}\{G, G$ is preopen set $\}=\operatorname{Int}_{P}(A)$. That is $H \subseteq \operatorname{Int}_{P}(A)$. Thus, $\operatorname{Int}_{P}(A)$ is the largest preopen set contained in $A$.

3. Let $A$ be a preopen set. Since $\operatorname{Int}_{P}(A)$ is largest preopen contained in $A$ and $A \subseteq A$, so $A \subseteq \operatorname{Int}_{P}(A)$. And since $\operatorname{Int}_{P}(A) \subseteq A$. Thus, $A=\operatorname{Int}_{P}(A)$.Conversely: It follows from $\operatorname{part}(1)$.

4. Let $U=\operatorname{Int}_{P}(A)$.So $U$ is preopen set, then $U=\operatorname{Int}_{P}(U)$ by (3). Thus, $\operatorname{Int}_{P}(A)=$ $\operatorname{Int}_{P}\left(\operatorname{Int}_{P}(A)\right)$.

5. If $x \in A \backslash D_{P}(X \backslash A)$, then $x \notin D_{P}(X \backslash A)$ and so there exists a pre-open set $V$ containing $x$ such that $V \cap(X \backslash A)=\phi$. Thus, $x \in V \subseteq A$ and hence $x \in \operatorname{Int}_{P}(A)$. This shows that $A \backslash D_{P}(X \backslash A) \subseteq \operatorname{Int}_{P}(A)$. Now let $x \in \operatorname{Int}_{P}(A)$. Since $\operatorname{Int}_{P}(A) \cap(X \backslash A)=\phi$, we have $x \notin D_{P}(X \backslash A)$. Therefore, $\operatorname{Int}_{P}(A)=A \backslash D_{P}(X \backslash A)$.

6. Using (4) and Theorem 3.5, we have $X \backslash \operatorname{Int}_{P}(A)=X \backslash\left(A \backslash D_{P}(X \backslash A)\right)=(X \backslash A) \cup$ $D_{P}(X \backslash A)=C l_{P}(X \backslash A)$.

7.Using (4) and Theorem 3.5, we have $\operatorname{Int}_{P}(X \backslash A)=(X \backslash A) \backslash D_{P}(A)=X \backslash(A \cup$ $\left.D_{P}(A)\right)=X \backslash C l_{P}(A)$.

8. Let $x \in \operatorname{Int}_{P}(A)$, then $x$ is pre-interior of $A$, so there exists a preopen set $V$ such that $x \in V \subseteq A$, but $A \subseteq B$. So $x \in V \subseteq B$, then $x$ is pre-interior of $B$. Hence, $x \in \operatorname{Int}_{P}(B)$. Thus $\operatorname{Int}_{P}(A) \subseteq \operatorname{Int}_{P}(B)$.It follows from part (8).

9. It follows from part (8).

The converse of (8) in Proposition 3.2. is not true in general as seen in the following example:

\section{Example 3.7}

Let $X=\{a, b, c, d, e\}$ and defined closure operator $c: P(X) \rightarrow P(X)$ by: 


$c(A)=\left\{\begin{array}{lc}A & \text { Journal of Garmian University } \\ \{b, e\} & \text { if } A \in\{\phi,\{a\},\{b, c, d, e\}\}=\mathcal{F} \\ \{a, b, e\} & \text { if } A \in\{\{b\},\{e\},\{b, e\}\}=\mathcal{G} \\ \{b, c, d, e\} & \text { if } A \in\{\{a, b\},\{a, e\},\{a, b, e\}\}=\mathcal{H} \\ & \text { if } A \notin\{\mathcal{F}, \mathcal{G}, \mathcal{H}\} \text { and } A \subseteq\{b, c, d, e\} \\ X & \text { otherwise }\end{array}\right.$

Let $A=\{a, b\}$ and $B=\{a, c, d\}$ be subsets of $X$. Then $\operatorname{Int}_{P}(A)=\{a\} \subseteq \operatorname{Int}_{P}(B)=$ $\{a, c, d\}$.

\section{Definition 3.6}

For a subset $\mathrm{A}$ of a closure space $(X, c)$, the set

(1) $B_{P}(A)=A \backslash \operatorname{Int}_{P}(A)$ is called the pre-border of $A$.

(2) $\operatorname{Fr}_{P}(A)=\operatorname{Cl}_{P}(A) \backslash \operatorname{Int}_{P}(A)$ is called the pre-frontier of $A$.

\section{Remark 3.1}

If $A$ is a preclosed subset of $\mathrm{X}$, then $B_{P}(A)=F r_{P}(A)$.

\section{Example 3.8}

Let $(X, c)$ be the closure space which is described in Example 3.7. Let $A=\{a, b, e\}$ be a subset of $X$. Then $\operatorname{Int}_{P}(A)=\{a\}$, and so $B_{P}(A)=\{b, e\}$. Since $A=\{a, b, e\}$ is preclosed, $C l_{P}(A)=\{a, b, e\}$ and thus $F_{P}(A)=\{b, e\}$.

\section{Example 3.9}

Consider the closure space $(X, c)$ which is given in Example 3.3. For a subset $A=\{b, c, d\}$ of $X$, we have $\operatorname{Int}_{P}(A)=\{c, d\}$ and $C l_{P}(A)=\{b, c, d, e\}$. Hence $B_{P}(A)=$ $\{b\}$ and $F r_{P}(A)=\{b, e\}$.

\section{Proposition 3.3}

For a subset $A$ of a closure space $(X, c)$, the following statements hold:

(1) $A=\operatorname{Int}_{P}(A) \cup B_{P}(A)$.

(2) $\operatorname{Int}_{P}(A) \cap B_{P}(A)=\phi$.

(3) $A$ is a preopen set if and only if $B_{P}(A)=\phi$.

(4) $B_{P}\left(\operatorname{Int}_{P}(A)\right)=\phi$.

(5) $\operatorname{Int}_{P}\left(B_{P}(A)\right)=\phi$.

(6) $B_{P}\left(B_{P}(A)\right)=B_{P}(A)$.

(7) $B_{P}(A)=A \cap C l_{P}(X \backslash A)$. 
(8) $B_{P}(A)=A \cap D_{P}(X \backslash A)$.

\section{Proof:}

\section{Obvious.}

2. Obvious.

3.It follows from Proposition 3.2 (3) and Definition 3.5 (1).

4. Since $\operatorname{Int}_{P}(A)$ is preopen, it follows from (3) that $B_{P}\left(\operatorname{Int}_{P}(A)\right)=\phi$.

5. If $x \in \operatorname{Int}_{P}\left(B_{P}(A)\right)$, then $x \in B_{P}(A) \subseteq A$, and then $x \in \operatorname{Int}_{P}(A)$. Thus, $x \in B_{P}(A) \cap$ $\operatorname{Int}_{P}(A)=\phi$, which is a contradiction. Hence, $\operatorname{Int}_{P}\left(B_{P}(A)\right)=\phi$.

6. Using (5), we get $B_{P}\left(B_{P}(A)\right)=B_{P}(A) \backslash \operatorname{Int}_{P}\left(B_{P}(A)\right)=B_{P}(A)$.

7.Using Proposition $3.2(6)$ we have $B_{P}(A)=A \backslash \operatorname{Int}_{P}(A)=A \backslash\left(X \backslash C l_{P}(X \backslash A)\right)=$ $A \cap C l_{P}(X \backslash A)$.

8. Applying (7) and Theorem 3.5, we have we have to show $C l_{P}(A) \subseteq A$. To this end, let $x \notin A$. Then $x \notin F r_{P}(A)$. So $x \notin C l_{P}(A) \backslash \operatorname{Int}_{P}(A)$. But since $\operatorname{Int}_{P}(A) \subseteq A$ and $x \notin$ $A$, so $x \notin C l_{P}(A)$. This means that, $C l_{P}(A) \subseteq A$. So $A$ is preclosed.

\section{Lemma 3.1}

For a subset $A$ of a closure space $(X, c), A$ is preclosed if and only if $\operatorname{Fr}_{P}(A) \subseteq A$.

\section{Proof.}

Assume that $A$ is preclosed. Then $\operatorname{Fr}_{P}(A)=C l_{P}(A) \backslash \operatorname{Int}_{P}(A)=A \backslash \operatorname{Int}_{P}(A) \subseteq A$. Conversely suppose that $\operatorname{Fr}_{P}(A) \subseteq A$, then $C l_{P}(A) \backslash \operatorname{Int}_{P}(A) \subseteq A$.To show $\mathrm{A}$ is preclosed.. In view of Theorem 3.3(7). We have to show $C l_{P}(A) \subseteq A$. To this end, let $x \notin A$. Then $x \notin F r_{P}(A)$. So $x \notin C l_{P}(A) \backslash \operatorname{Int}_{P}(A)$. But since $\operatorname{Int}_{P}(A) \subseteq A$ and $x \notin$ $A$, so $x \notin C l_{P}(A)$. This means that, $C l_{P}(A) \subseteq A$. So $A$ is preclosed.

\section{Theorem 3.8}

For a subset $A$ of a closure space $(X, c)$, the following assertions are valid:

(1) $C l_{P}(A)=\operatorname{Int}_{P}(A) \cup \operatorname{Fr}_{P}(A)$.

(2) $\operatorname{Int}_{P}(A) \cap \operatorname{Fr}_{P}(A)=\emptyset$.

(3) $B_{P}(A) \subseteq F r_{P}(A)$.

(4) $\operatorname{Fr}_{P}(A)=B_{P}(A) \cup\left(D_{P}(A) \backslash \operatorname{Int}_{P}(A)\right)$.

(5) $A$ is a preopen set if and only if $\operatorname{Fr}_{P}(A)=B_{P}(X \backslash A)$.

(6) $\operatorname{Fr}_{P}(A)=C l_{P}(A) \cap C l_{P}(X \backslash A)$.

(7) $\operatorname{Fr}_{P}(A)=F r_{P}(X \backslash A)$.

(8) $\operatorname{Fr}_{P}(A)$ is preclosed. 
(9) $\operatorname{Fr}_{P}\left(F r_{P}(A)\right) \subseteq \operatorname{Fr}_{P}(A)$.

(10) $\operatorname{Fr}_{P}\left(\operatorname{Int}_{P}(A)\right) \subseteq F r_{P}(A)$.

(11) $\operatorname{Fr}_{P}\left(C l_{P}(A)\right) \subseteq \operatorname{Fr}_{P}(A)$.

(12) $\operatorname{Int}_{P}(A)=A \backslash F r_{P}(A)$.

\section{Proof:}

\section{Obvious.}

2. Obvious.

3. Obvious.

4. Using Theorem 3.5, we obtain $F_{P}(A)=C l_{P}(A) \backslash \operatorname{Int}_{P}(A)=\left(A \cup D_{P}(A)\right) \cap$ $\left(X \backslash \operatorname{Int}_{P}(A)\right)=\left(A \backslash \operatorname{Int}_{P}(A)\right) \cup\left(D_{P}(A) \backslash \operatorname{Int}_{P}(A)\right)=B_{P}(A) \cup\left(D_{P}(A) \backslash \operatorname{Int}_{P}(A)\right)$.

5. Assume that $A$ is preopen. Then $F_{P}(A)=B_{P}(A) \cup\left(D_{P}(A) \backslash \operatorname{Int}_{P}(A)\right)=\phi \cup$ $\left(D_{P}(A) \backslash A\right)=D_{P}(A) \backslash A=B_{P}(X \backslash A)$ by using (4), Proposition 3.3 (3), Proposition 3.2 (3) and Proposition 3.3 (8).

Conversely; suppose that $F_{P}(A)=B_{P}(X \backslash A)$. Then $\phi=F r_{P}(A) \backslash B_{P}(X \backslash A)=$ $\left(C l_{P}(A) \backslash \operatorname{Int}_{P}(A)\right) \backslash\left((X \backslash A) \backslash \operatorname{Int}_{P}(X \backslash A)\right)=A \backslash \operatorname{Int}_{P}(A)=B_{P}(A)$. By (5) and (6) of Proposition 3.2, and so by Proposition 3.3(3), $A$ is preopen.

6.It follows from Proposition 3.2(6).

7.It is followed from (6).

8. we have $C l_{P}\left(\operatorname{Fr}_{P}(A)\right)=C l_{P}\left(C l_{P}(A) \cap C l_{P}(X \backslash A)\right) \subseteq C l_{P}\left(C l_{P}(A)\right) \cap C l_{P}\left(C l_{P}(X \backslash\right.$ $A))=C l_{P}(A) \cap C l_{P}(X \backslash A)=F_{P}(A)$. Obviously $\operatorname{Fr}_{P}(A) \subseteq C l_{P}\left(F_{P}(A)\right)$, and so $\operatorname{Fr}_{P}(A)=\operatorname{Cl}_{P}\left(\operatorname{Fr}_{P}(A)\right)$. Hence $\operatorname{Fr}_{P}(A)$ is preclosed.

9. This is by (8) and Lemma 3.1 .

10. Proposition $3.2(4)$, we get $F_{P}\left(\operatorname{Int}_{P}(A)\right)=C l_{P}\left(\operatorname{Int}_{P}(A)\right) \backslash \operatorname{Int}_{P}\left(\operatorname{Int}_{P}(A)\right) \subseteq$ $C l_{P}(A) \backslash \operatorname{Int}_{P}(A)=\operatorname{Fr}_{P}(A)$.

11. We obtain $F_{P}\left(C l_{P}(A)\right)=C l_{P}\left(C l_{P}(A)\right) \backslash \operatorname{Int}_{P}\left(C l_{P}(A)\right) \subseteq C l_{P}(A) \backslash \operatorname{Int}_{P}(A)=$ $\operatorname{Fr}_{P}(A)$.

12. We get $A \backslash \operatorname{Fr}_{P}(A)=A \backslash\left(C l_{P}(A) \backslash \operatorname{Int}_{P}(A)\right)=A \cap\left(\left(X \backslash C l_{P}(A)\right) \cup \operatorname{Int}_{P}(A)\right)=\phi \cup$ $\left(A \cup \operatorname{Int}_{P}(A)\right)=\operatorname{Int}_{P}(A)$.

The converse of (3) is not true in general as seen in the following example.

\section{Example 3.10}

Example 3.9 shows that the reverse inclusion of Theorem 3.9 (3) is not valid in general. 


\section{Definition 3.7}

For a subset $A$ of a closure space $(X, c)$, the pre-interior of $X \backslash A$ is called the preexterior of $A$, and it is denoted by $\operatorname{Ext}_{P}(A)$, that is, $\operatorname{Ext}_{P}(A)=\operatorname{Int}_{P}(X \backslash A)$.

\section{Example 3.11}

Consider the closure space $(X, c)$ which is given in Example 3.7. For subsets $A=\{a, b, c\}$ and $B=\{b, d\}$ of $X$, we have $\operatorname{Ext}_{P}(A)=\{d, e\}$ and $\operatorname{Ext}_{P}(B)=\{a, c, e\}$.

\section{Theorem 3.9}

For subsets $A$ and $B$ of a closure space $(X, c)$, the following assertions are valid.

(1) $\operatorname{Ext}_{P}(A)$ is preopen.

(2) $\operatorname{Ext}_{P}(A)=X \backslash C l_{P}(A)$.

(3) $\operatorname{Int}_{P}(A) \subseteq \operatorname{Int}_{P}\left(C l_{P}(A)\right)=\operatorname{Ext}_{P}\left(\operatorname{Ext}_{P}(A)\right)$.

(4) If $A \subseteq B$ then $\operatorname{Ext}_{P}(B) \subseteq \operatorname{Ext}_{P}(A)$.

(5) $\operatorname{Ext}_{P}(A \cup B) \subseteq \operatorname{Ext}_{P}(A) \cap \operatorname{Ext}_{P}(B)$.

(6) $\operatorname{Ext}_{P}(A) \cup \operatorname{Ext}_{P}(B) \subseteq \operatorname{Ext}_{P}(A \cap B)$.

(7) $\operatorname{Ext}_{P}(X)=\phi, \operatorname{Ext}_{P}(\phi)=X$.

(8) $\operatorname{Ext}_{P}(A)=\operatorname{Ext}_{P}\left(X \backslash E x t_{P}(A)\right)$.

(9) $X=\operatorname{Int}_{P}(A) \cup \operatorname{Ext}_{P}(A) \cup \operatorname{Fr}_{P}(A)$.

\section{Proof.}

1.It follows from Lemma 3.1 and Proposition 3.2(1).

2.It is straightforward by Proposition 3.2(7).

3. Applying (6) and (8) of Proposition 3.2, we get $\operatorname{Ext}_{P}\left(\operatorname{Ext}_{P}(A)\right)=\operatorname{Ext}_{P}\left(\operatorname{Int}_{P}(X \backslash\right.$ $A))=\operatorname{Int}_{P}\left(X \backslash \operatorname{Int}_{P}(X \backslash A)\right)=\operatorname{Int}_{P}\left(C l_{P}(A)\right) \supset \operatorname{Int}_{P}(A)$.

4. Assume that $A \subset B$, then $\operatorname{Ext}_{P}(B)=\operatorname{Int}_{P}(X \backslash B) \subseteq \operatorname{Int}_{P}(X \backslash A)=\operatorname{Ext}_{P}(A)$ by using Proposition 3.2(8).

5. Applying Proposition 3.2 (10), we get $\operatorname{Ext}_{P}(A \cup B)=\operatorname{Int}_{P}(X \backslash(A \cup B))=$ $\operatorname{Int}_{P}((X \backslash A) \cap(X \backslash B)) \subseteq \operatorname{Int}_{P}(X \backslash A) \cap \operatorname{Int}_{P}(X \backslash B)=\operatorname{Ext}_{P}(A) \cap \operatorname{Ext}_{P}(B)$.

6. Using Proposition 3.2 (9), we obtain $\operatorname{Ext}_{P}(A \cap B)=\operatorname{Int}_{P}(X \backslash(A \cap B))=$ $\operatorname{Int}_{P}\left((X \backslash A) \cup(X \backslash B) \supseteq \operatorname{Int}_{P}(X \backslash A) \cup \operatorname{Int}_{P}(X \backslash B)=\operatorname{Ext}_{P}(A) \cup \operatorname{Ext}_{P}(B)\right.$.

7. $\operatorname{Ext}_{P}(X)=\operatorname{Int}_{P}(X \backslash X)=\operatorname{Int}_{P}(\phi)=\phi$. Also $\operatorname{Ext}_{P}(\phi)=\operatorname{Int}_{P}(X \backslash \phi)=\operatorname{Int}_{P}(X)=X$.

8. Using Proposition 3.2 (4), we have $\operatorname{Ext}_{P}\left(X \backslash E x t_{P}(A)\right)=\operatorname{Ext}_{P}\left(X \backslash \operatorname{Int}_{P}(X \backslash A)\right)=$ $\operatorname{Int}_{P}(X \backslash A)=\operatorname{Ext}_{P}(A)$. 
9.

$\operatorname{Int}_{P}(A) \cup \operatorname{Ext}_{P}(A) \cup \operatorname{Fr}_{P}(A)=X \backslash \operatorname{Fr}_{P}(A) \cup F r_{P}(A)=X$.

\section{Example 3.12}

Let $(X, c)$ be a closure space which is given in Example 3.7. Let $A=\{b, e\}$ and $B=\{c, d, e\}$. Then $\operatorname{Ext}_{P}(B)=\{a\} \subseteq\{a, c, d\}=\operatorname{Ext}_{P}(A)$. This shows that the converse of (4) in Theorem 3.9 is not valid. Now let $A=\{d, e\}$ and $B=\{c\}$. Then $\operatorname{Ext}_{P}(A \cup B)=\{a\} \neq\{a, b\}=\{a, b, c\} \cap\{a, b, d, e\}=\operatorname{Ext}_{P}(A) \cap \operatorname{Ext}_{P}(B)$ which shows that the equality in Theorem 3.9 (5) is not valid. Finally let $A=\{a, b\}$ and $B=\{c, d, e\}$. Then $\operatorname{Ext}_{P}(A \cap B)=\{a, b, c, d, e\}$ and $\operatorname{Ext}_{P}(A) \cup \operatorname{Ext}_{P}(B)=\{a, c, d, e\}$. This shows that the equality in Theorem $3.9(6)$ is not valid.

\section{Theorem 3.10}

Let $(X, c)$ be idempotent closure space and $A, B \subseteq X$, if $A \subseteq B$, then $i(A) \subseteq i(B)$.

\section{Proof.}

Since $A \subseteq B$, then $X \backslash B \subseteq X \backslash A$, so $c(X \backslash B) \subseteq c(X \backslash A)$ ( since $c$ is closure operator), then $X \backslash c(X \backslash A) \subseteq X \backslash c(X \backslash B)$, so $i(A) \subseteq i(B)$.

\section{Theorem 3.11}

Let $(X, c)$ be idempotent closure space and $A \subseteq X$, then $i(i(A))=i(A)$.

Proof. Let $i(A)=X \backslash c(X \backslash A)$, so $i(i(A))=i(X \backslash c(X \backslash A))=X \backslash c(X \backslash(X \backslash c(X \backslash A)))=$ $X \backslash c(X \backslash(X \backslash c(X \backslash A)))=X \backslash c(c(X \backslash A)))=X \backslash c(c(X \backslash A)))=X \backslash c(c(X \backslash A)))=$ $X \backslash c(X \backslash A)=i(A)$.

\section{References}

1. Khampakdee, J., Semi-open sets in closure spaces. Ph. D. thesis, BRNO University of technology, 2009.

2. J.Khampakdee, Semi-open sets in biclosure spaces. Discussiones Mathematicae, General Algebra-accepted..

3. Mashhour, A.S., M.E.A. El-Monesf, and S.N. El-Deeb, On pre-continuous and weak pre continuous mappings. Proc. Math. Phys. Soc. Egypt 53, 1982: p. 47-53.

4. Darwesh, H.M., Almost-continuity, Pre-continuity and Preopen sets in Closure Spaces. (submit).

5. Ahmad, B. and S. Hussain, Properties of $\gamma$-Operations on Topological Spaces. Aligarh Bull.Math, 2003. 1(22): p. 45-51. 
6. B.Khalaf, A. and S.F. Namiq, Generalized lammda-Closed Sets and (Lammda,Gamma) ${ }^{\wedge} *_{-}$Continuous Functions International Journal of Scientific \& Engineering Research, 2012. 3(12).

7. B.Khalaf, A. and S.F. Namiq, Generalized Lammda-Closed Sets and (Lammda,Gamma)^*-Continuous Journal of Garmyan University, 2017: p. 23100087.

8. $\quad$ B.Khalaf, A. and S.F. Namiq, $\lambda \_\beta c$-Connected Spaces and $\lambda \_\beta c$-Components. Journal of Garmyan University, 2017.

9. B.Khalaf, A. and S.F. Namiq, ON Minimal $\lambda^{\wedge} *_{-}$Open Sets, . International Journal of Scientific \& Engineering Research, October-2014. 5(10).

10. Darwesh, H.M. and S.F. Namiq, On Minimal $\lambda \_\beta c$-Open Sets Journal of Garmyan University, 2017.

11. Darwesh, H.M., S.F. Namiq, and W.K. Kadir, Maximal $\lambda c-O p e n$ Sets. ICNS-2016, 2017.

12. F.Namiq, S., Lammda sub beta c-Open Sets and Topological Properties. Journal of Garmyan University, 2017(2310-0087).

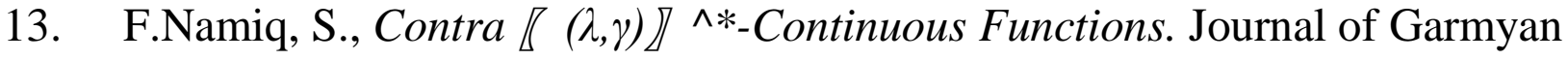
University, 2017.

14. Hussain, S. and B. Ahmad, On Minimal $\gamma$-Open Sets. Eur. J. Pure Appl. Maths, 2009. 3(2): p. 338-351.

15. Kasahara, S., Operation-Compact Spaces. Math. Japon, 1979(24): p. 97-105.

16. Khalaf, A.B., H.M. Darwesh, and S.F. Namiq, $\lambda \_c$-Connected Space Via $\lambda \_c$-Open Sets. Journal of Garmyan University, 2017.

17. Khalaf, A.B. and S.F. Namiq, New types of continuity and separation axiom based operation in topological spaces. M. Sc. Thesis, University of Sulaimani, 2011.

18. Khalaf, A.B. and S.F. Namiq, Lammda sub c-Open Sets and Lammda sub cSeparation Axioms in Topological Spaces. Journal of Advanced Studies in Topology, 2013. 4(1): p. 150-158.

19. Namiq, S.F., Lammda-RO and Lammda-R1 Spaces. Journal of Garmyan University, 2014. 4(3).

20. Namiq, S.F., $\lambda$ sc-open sets and topological properties. Journal of Garmyan University, 2014.

21. Namiq, S.F., $\lambda$-Connected Spaces Via $\lambda$-Open Sets. Journal of Garmyan University, 2015.

22. Namiq, S.F., $\lambda$ _sc-Connected Spaces Via $\lambda \_s c-O p e n$ Sets. Journal of Garmyan University, 2017. 
23. Namiq, S.F., Generalized $\lambda \_c$-Open Set. International Journal of Scientific \& Engineering Research, June-2017. 8(6).

24. Namiq, S.F., $\lambda \_c$-Separation Axioms Via $\lambda \_c$-open sets. International Journal of Scientific \& Engineering Research May-2017. 8.

25. Namiq, S.F., Lammda sub ac-Open Sets and Topological Properties. pre-print.

26. Ogata, H., Operations on Topological Spaces and Associated Topology. Math. Japon, 1991. 36(1): p. 175-184.

27. Namiq, S.F., On Minimal $\lambda \_b c$-Open Sets Journal of Garmyan University, 2018accepted.

28. Namiq, S.F., On Minimal $\lambda \_\alpha c$-Open Sets Journal of Garmyan University, 2018accepted.

29. Sharma, J.N ”Topology”,. Krishna Prakashna Mandir, India., 1977.

30. Sharma, J.N. and J.P. Chauhan, "Topology(General and Algebraic”. Krishna Prakashna Media, India, 2011. 